\title{
Orthogonal Pure States in Operator Theory
}

\author{
Jan Hamhalter
}

\begin{abstract}
We summarize and deepen existing results on systems of orthogonal pure states in the context of JB algebras and $C^{*}$-algebras. Especially, we focus on noncommutative generalizations of some principles of topology of locally compact spaces such as exposing points by continuous functions, separating sets by continuous functions, and multiplicativity of pure states.
\end{abstract}

\section{Introduction and preliminaries}

The aim of this paper is to summarize and deepen recent results on the systems of orthogonal pure states in operator theory and comment on their relations to axiomatic foundations of quantum mechanics. There are two main motivations for our study of pure states and their orthogonal systems. At first, in the standard axiomatics of quantum mechanics pure states of a physical system are identified with the rays in a separable Hilbert space. In this approach orthogonal pure states, embodying mutually exclusive states of the system, correspond to orthogonal one-dimensional subspaces. For this reason, the systems of pure states have been studied in connection with fundamental questions of quantum mechanics such as hidden variables problem, description of independence of quantum system, etc. Secondly, from the mathematical standpoint, pure states encode much of the structure of operator algebras. Any commutative $C^{*}$-algebra $\mathcal{A}$ may be identified with the algebra of complex continuous functions on a locally compact space $X$, while pure states correspond to points of $X$. There are many important principles on separating points and sets in topology of locally compact spaces, as for example the Uryshon Lemma and others. The objective of this note is to overview possible extensions of various principles of this kind to the realm of the $C^{*}$-algebras and the Jordan-Banach algebras.

As the starting point we recall basic properties of commutative operator algebras. Any commutative $C^{*}$-algebra is isomorphic to the algebra $C_{0}(X)$ of all continuous complex functions on a locally compact Hausdorff space $X$ vanishing at infinity. Associative JB algebras are nothing but algebras of all real functions from $C_{0}(X)$. At this level there is no substantial difference between the Jordan algebras and the $C^{*}$-algebras. One of the classical results of Banach function algebras says that a state $\varrho$ on $C_{0}(X)$ is pure if and only if it is multiplicative, i.e. precisely when $\varrho(f g)=\varrho(f) \varrho(g)$ for all $f, g \in C_{0}(X)$. Moreover, there is a 
one-to-one correspondence between the pure states and the points of $X$ in the sense that every pure state $\varrho$ is of the form $\varrho(f)=f(x),\left(f \in C_{0}(X)\right)$, where $x \in X$. These results are far from being true in the noncommutative case. In fact, all pure states on a $C^{*}$-algebra are multiplicative exactly when this algebra is commutative. However, we shall establish that even in the noncommutative case pure states are multiplicative on large parts of operator algebras.

Another fact concerning exposing point by a continuous function will be dealt with in more general noncommutative setting. If the compact space $X$ is metrizable, then any point $x \in X$ can be exposed by a continuous function $f$ on $X$ with values in $[0,1]$, meaning that $x$ is the only point such that $f(x)=$ 1. Among others we will show that this principle remains valid for separable algebras.

Finally, working in locally compact space $X$, it is easy to verify that given a sequence $\left(x_{n}\right)$ discrete as a subspace of $X$, we can always find a sequence $\left(f_{n}\right)$ of continuous function on $X, 0 \leq f_{n} \leq 1$, having pairwise disjoint supports and satisfying $f_{n}\left(x_{m}\right)=\delta_{n m}$. In other words, points can be separated by functions. We will investigate to what extend can this fact be extended to nonassociative generalization of compact Hausdorff spaces given by JB algebras.

What makes the problems outlined above interesting and difficult is the fact that "points" and "sets" cannot be localized in one space. Namely, in general operator setting points correspond to elements of the dual spaces while open sets correspond to special projection in the double duals.

Let us now recall basic notions and fix the notation. Our basic structure will be JB algebra. A $J B$ algebra is a real Banach algebra $(A, \circ)$ such that for all $a, b \in A$, (i) $a \circ b=b \circ a$, (ii) $(a \circ b) \circ a^{2}=a \circ\left(b \circ a^{2}\right)$, (iii) $\left\|a^{2}\right\|=\|a\|^{2}$, (iv) $\left\|a^{2}+b^{2}\right\| \geq\|a\|^{2}$. For all unmentioned details on operator algebras we refer to monographs [10, 12, 17, 18, 20]. In what follows $A$ will stand for a JB algebra and $B(H)$ for the algebra of all bounded operators acting on a Hilbert space $H$. An important example of a JB algebra is the algebra of all selfadjoint operators of a $C^{*}$-algebra $\mathcal{A}$ endowed with the product $a \circ b=1 / 2(a b+b a)$. A state on a JB algebra $A$ is a norm one functional $\varrho$ on $A$ such that $\varrho\left(a^{2}\right) \geq 0$ for all $a \in A$. The pure state is an extreme point of the convex set of all states. Two canonical examples of pure states will be important for us. At first, the Dirac measures $\delta_{x}, x \in X$, defined on the function algebra $C_{0}^{R}(X)$ of all real continuous functions on a locally compact space $X$ vanishing at infinity by $\delta_{x}(f)=f(x),\left(f \in C_{0}(X)\right.$, are the only pure states on $C_{0}^{R}(X)$. Secondly, the vector state on the self-adjoint part of a $C^{*}$-algebra $\mathcal{A}$ acting on a Hilbert space $H$ associated with a unit vector $\xi \in H$ by the formula $\omega_{\xi}(a)=(a \xi, \xi)$ $(a \in \mathcal{A})$ is a pure state that can be considered as the noncommutative version of the Dirac measure. Pure states $\varrho_{1}$ and $\varrho_{2}$ on $A$ are called orthogonal if they have the maximal possible distance, i.e. if $\left\|\varrho_{1}-\varrho_{2}\right\|=2$. The vector states on the algebra of all self-adjoint operators on a given Hilbert space are orthogonal exactly when the corresponding vectors are orthogonal. In case of associative algebras all distinct pure states are orthogonal. It is a key property of the quantum model that there are distinct but not orthogonal pure states (see Bohr's concept of complementarity). 


\section{Individual pure state}

The problem of exposing point of a compact space by a continuous function has given impetus to the following definition. An element $a \in A,\|a\| \leq 1, a \geq 0$, is determining for a pure state $\varrho$ on $A$ if $\varrho(a)=1$ and $\varphi(a)<1$ for any pure state $\varphi$ on $A$ different from $\varrho$. In the physical terminology, the state $\varrho$ admits the determining element if and only if there is some observable $O$ with values in $[0,1]$ such that the system is in the state $\varrho$ precisely when we obtain value 1 when measuring $O$. As an example, an atomic projection of a Hilbert space $H$ onto one-dimensional subspace spanned by the unit vector $\xi \in H$ is a determining element for the vector state $\omega_{\xi}$ on the algebra of all self-adjoint operators on a Hilbert space $H$. This is a typical situation in the standard formalism of quantum mechanics. The following proposition describes states admitting determining elements:

2.1. Proposition. (15]) If $A$ is a unital algebra then a pure state $\varrho$ on $A$ admits a determining element if and only if its left ideal $L_{\varrho}=\left\{\varrho\left(x^{2}\right)=0 \mid x \in A\right\}$ has a countable approximate unit. Especially, any pure state on a separable $J B$ algebra has a determining element.

The proof is based on the analysis of approximate units and pure states. This result is far from being true for nonseparable algebra. Indeed, in contrast to Proposition 2.1, only normal pure states on JBW algebras qualify for having

determining elements. Let us recall that a JB algebra is called a JBW algebra if it is a dual Banach space. A state on JBW algebra is called normal if it can be identified with element from the predual.

2.2. Theorem. (15]) A pure state $\varrho$ on a JBW algebra $M$ has a determining element if and only if $\varrho$ is normal. Especially, if $M$ is a von Neumann algebra then $\varrho$ has a determining element if and only if it is a vector state concentrated on a direct summand isomorphic to $B(H)$.

The previous Theorem says that the states described by the rays in a Hilbert space $H$ are the only pure states on von Neumann algebras having determining elements. This advocates basic assumptions in early models of quantum mechanics.

\section{Orthogonal pure states and supporting sys- tems}

In this section we shall deal with a more complicated case of a system of orthogonal pure states. Suppose that $\left(\varrho_{n}\right)$ is a sequence of (pairwise) orthogonal pure states on $A$. A sequence $\left(b_{n}\right)$ in $A$ with $0 \leq b_{n} \leq 1$, and $b_{n} \circ b_{m}=0$ whenever 
$n \neq m$ is called supporting for $\left(\varrho_{n}\right)$ if

$$
\varrho_{n}\left(b_{m}\right)=\delta_{m n} \quad \text { for all } n, m .
$$

If, in addition, each $b_{n}$ is determining for $\varrho_{n}$, we call $\left(b_{n}\right)$ a determining supporting sequence. In example of associative algebra in the introduction we have seen that a sequence of orthogonal pure states has a supporting sequence if the corresponding points can be separated by open sets. In order to find an analogy of this result for nonassociative algebras we shall need the following concepts. Let $A$ be canonically embedded into its second dual $A^{* *}$. It is known that $A^{* *}$ is a JBW algebra extending the product in $A$. A projection $p$ in $A^{* *}$ is called open if there is an increasing net of elements $\left(a_{\lambda}\right)$ in $A$ with $a_{\lambda} \nearrow p$. A projection $p$ in $A^{* *}$ is closed if $1-p$ is open. Any state $\varrho$ on $A$ canonically extends to a normal state on $A^{* *}$. Hence, there is a smallest projection $s(\varrho)$ in $A^{* *}$ such that $\varrho(s(\varrho))=1$. We call $s(\varrho)$ the support projection of $\varrho$. It is known that $s(\varrho)$ is always minimal and closed if $\varrho$ is pure. It was proved in 14 that a system $\varrho_{\alpha}$ of orthogonal pure states admits a supporting system if and only if there is a system $\left(p_{\alpha}\right)$ of open, pairwise orthogonal projections in $A^{* *}$, such that $s\left(\varrho_{\alpha}\right) \leq p_{\alpha}$ for each $\alpha$. This is a precise analogy of the classical case. Our main result gives sufficient conditions for the existence of supporting system in terms of primitive ideal space. Let $P(A)$ be the set of all pure states on $A$ equipped with the weak*topology. Let $c(\varrho)$ be the central cover of $\varrho$, i.e. the smallest central projection in $A^{* *}$ majorizing the support projection $s(\varrho)$. We define a homomorphism $\pi_{\varrho}: A \rightarrow A^{* *}$ by $\pi_{\varrho}(x)=c(\varrho) \circ x, x \in A$. In case of $C^{*}$-algebras $\pi_{\varrho}$ corresponds to the G.N.S. representation of $\varrho$. The primitive ideal space is now defined as the set of all kernels of the representations, $\operatorname{Prim}(A)=\left\{\operatorname{Ker} \pi_{\varrho} \mid \varrho \in P(A)\right\}$. The primitive ideal space is endowed with the Jacobson topology whose closure operation is $S \subset \operatorname{Prim}(A) \rightarrow \bar{S}=\{F \in \operatorname{Prim}(A) \mid F \supset \cap S\}$. The canonical map $\tau$ now sends pure state $\varrho$ to its kernel $\operatorname{Ker} \varrho$. A set $P$ in the pure state space $P(A)$ is called almost separated if its image $\tau(P)$ in $\operatorname{Prim}(A)$ can be covered by disjoint open sets $\left(U_{\alpha}\right)$ such that each open set $\tau^{-1}\left(U_{\alpha}\right)$ contains finitely many elements. The following result says that any almost separated sequence of orthogonal pure states admits a supporting sequence.

3.1. Theorem. (14]) Any sequence $\left(\varrho_{n}\right)$ of almost separated orthogonal pure states on a JB algebra $A$ has a supporting sequence. This sequence can be chosen determining if $A$ is separable.

By combining the Hahn-Banach and the Krein-Milman theorems we can derive the following consequences of the previous results for the restriction properties of states. By Theorem 3.1 any finite sequence $\varrho_{1}, \varrho_{2}, \ldots \varrho_{n}$ of orthogonal pure states on a separable algebra $A$ has a determining sequence $b_{1}, b_{2}, \ldots, b_{n}$. The algebra $C$ generated by $b_{1}, b_{2}, \ldots, b_{n}$ is associative and all states $\varrho_{1}, \varrho_{2}, \ldots, \varrho_{n}$ restrict simultaneously to pure states on $C$ and are uniquely given by these restrictions. If $n=2$ then the algebra $C$ can even be specified to be singly generated. From the physical point of view, two mutually exclusive 
states of the system are given by the structure of just one observable. Since any associative subalgebra can be extended to maximal one, we infer that any sequence $\left(\varrho_{n}\right)$ of almost separated states on a separable algebra admit a determining maximal associative subalgebra in the sense that each pure state $\varrho_{n}$ is uniquely determined by its restriction to this algebra.

The situation concerning infinitely many orthogonal pure states is discussed in the following Theorem. Following 3] we say that a sequence $\left(\varrho_{n}\right)$ of pure states on a JB algebra $A$ approaches to infinity if $\lim _{n \rightarrow \infty} \varrho_{n}(a)=0$ for every $a \in A$ such that the spectrum of $a$ contains zero. For example, if $A$ is the real part of the algebra of all continuous functions on the real line vanishing at infinity, then $\left(\delta_{x_{n}}\right)$ approaches to infinity if and only if $\left|x_{n}\right| \rightarrow \infty$.

3.2. Theorem. Let $A$ be a separable JB algebra and $\left(\varrho_{n}\right)$ be a sequence of orthogonal pure states approaching to infinity. The following statements are equivalent:

(i) $\left(\varrho_{n}\right)$ has a supporting system.

(ii) $\left(\varrho_{n}\right)$ has a determining supporting system.

(iii) There exists a maximal associative subalgebra $C$ of $A$ determining for $\left(\varrho_{n}\right)$.

(iv) There exists a maximal associative subalgebra $C$ of $A$ such that $\left(\varrho_{n} \mid C\right)$ forms an orthogonal sequence of pure states.

Proof: The equivalence of (i) and (ii) is the content of [14 Proposition 3.1] (ii) $\Rightarrow$ (iii) Suppose that $\left(b_{n}\right)$ is a supporting determining sequence for $\left(\varrho_{n}\right)$. The algebra $C^{\prime}$ generated by $b_{n}^{\prime} s$ is associative. Let $C$ be any maximal associative subalgebra extending $C$. Any state $\varrho_{n} \mid C^{\prime}$ is pure and extends uniquely to a pure state on $A$. Hence, state $\varrho_{n} \mid C^{\prime}$ has a unique extension to a state $\varrho_{n} \mid C$ which has to be, by the Krein-Milman theorem, a pure state. Hence, each $\varrho_{n}$ is uniquely determined by its pure restriction to $C$.

(iii) $\Rightarrow$ (iv) Let $C$ be an associative subalgebra fulfilling (iii). Since $\varrho_{n}$ 's have to be distinct on $C$ we have $\left\|\left(\varrho_{n}-\varrho_{m}\right) \mid C\right\|=2$ because $C$ is associative.

(iv) $\Rightarrow$ (i) Applying Theorem 3.1 to a separable subalgebra $C$ we get a desired supporting sequence for $\left(\varrho_{n}\right)$. The proof is completed.

These extension results contribute to the line of research in works [2, 4, [5, 7. 8.

\section{Multipicativity of orthogonal pure states}

As we have seen the pure state on an operator algebra is seldom multiplicative. On the other hand, it can be uniquely determined by multiplicative state on some maximal associative subalgebra provided that the algebra is separable. As it was shown by Akemann this does not hold for nonseparable algebras [2] 
However, we can still ask whether there exists some maximal associative subalgebra such that given pure state is multiplicative on it. The following problem, that is central for this section, seems to be a natural operator-theoretic extension of the equivalence between pureness and multiplicativity for states on function algebras.

4.1. Problem. Is a given sequence of orthogonal pure states on a JB algebra (resp. $C^{*}$-algebra) pure (i.e. multiplicative) on some maximal associative (resp. abelian) subalgebra?

There are many partial positive results along this line. E.Stormer haracterized pure states in terms of definite subasets [19. Aarnes and Kadison answered the problem in the positive for a pure state on a separable unital $C^{*}$-algebra [1. Then Akemann extended their result to finitely many states on a separable (not necessarily) unital $C^{*}$-algebra 2]. It was Barnes who realized that what is important is the separability of the G.N.S. representation of a given state. He established the positive answer for individual pure state with separable representation [7]. Also he gave, independently on Bunce [8], positive answer for pure states on Type I $C^{*}$-algebras [7]. The topic was then revived by Akemann, Anderson and Pedersen in the paper 4] dealing with states approaching to infinity. They have proved that pure orthogonal sequence of nearly inequivalent states on $C^{*}$-algebra approaching to infinity restrict simultaneously to multiplicative states on some maximal abelian subalgebra. Unfortunately, they needed additional assumption on accumulation points of this sequence. Despite these positive results we have proved that the answer to Problem 4.1 is in the negative. Moreover, it turns out that the counterexample is quite generic.

4.2. Counterexample. (16]) Let $\mathcal{A}$ be a separable unital infinitely dimensional $C^{*}$-algebra acting irreducibly on a Hilbert space $H$. Let $K$ be the algebra of all compact operators acting on $H$. Suppose that $\mathcal{A} / \mathcal{A} \cap K$ is noncommutative. Then there exists a sequence of orthogonal pure states on $\mathcal{A}$ that do not restrict (simultaneously) to pure states on any maximal commutative $C^{*}$-subalgebra of $\mathcal{A}$.

Proof: The irreducible algebra $\mathcal{A}$ either contains all compact operators or $\mathcal{A} \cap K=\{0\}$. The proof of both cases is given in [16. Here we present a simpler version of arguments concerning the case when $\mathcal{A} \cap K=\{0\}$. Since $H$ is separable we can find a dense sequence $\left(x_{n}\right)$ in its unit sphere. It means that the convex hull of the sequence $\left(\omega_{x_{n}}\right)$ of the corresponding vector states is weak*-dense in the state space of $A$ (see e.g. [17]). Set

$$
f=\sum_{n} \frac{1}{2^{n}} \omega_{x_{n}}
$$

By the improved Glimm's lemma [4, 11] there is an orthonormal basis $\left(\xi_{n}\right)$ of 
$H$ such that

$$
f(a)=\lim _{n \rightarrow \infty}\left(a \xi_{n}, \xi_{n}\right) .
$$

By taking the corresponding pure states $\left(\omega_{\xi_{n}}\right)$ we obtain a sequence of pure orthogonal states on $\mathcal{A}$ ( $\mathcal{A}$ is irreducible). Suppose that $C$ is a maximal abelian subalgebra such that all states $\left(\omega_{\xi_{n}}\right)$ are multiplicative on $C$. Then, obviously, $f$ is also multiplicative on $C$. In other words $f$ is pure on $C$ and so $f=\omega_{\xi_{n}}$ for all $n$ on $C$. By the density of the convex hull of $\omega_{x_{n}}$, we see that all states on $C$ are multiples of $f$. By the spectral theory $C$ has to be one-dimensional and so $\operatorname{dim} \mathcal{A}=1-$ a contradiction with irreducibility of $\mathcal{A}$ on an infinite-dimensional $H$.

The case when $\mathcal{A}$ contains the ideal of compact operators is based on the Weyl-von Neumann theorem and may be found in [16.

This counterexample has interesting consequence for the Calcin algebra, i.e. for the quotient of $B(H)$ by the ideal of compact operators $K$. Namely, we can construct a separable subalgebra of the Calcin algebra having a sequence of orthogonal pure states that are not simultaneously multiplicative on any maximal commutative $C^{*}$-algebra. In contrast to this, Anderson proved in [6] that for any sequence of states on the Calcin algebra there is a maximal associative subalgebra such that all members of this sequence are multiplicative on it. This indicates that the interplay between the size of $C^{*}$-algebra and the size of its maximal abelian subalgebras is quite delicate.

Counterexample 4.2 indicates possible limitations to improvements of positive results. We have to put some additional conditions on the system of orthogonal pure states as it is shown in the following theorem.

4.3. Theorem. (16]) Let $\left(\varrho_{n}\right)$ be a sequence of orthogonal pure states on a JB algebra $A$ approaching to infinity and such that $c\left(\varrho_{n}\right) A^{* *}$ is $\sigma$-finite for all $n$. Suppose further that $\sum_{n=m}^{\infty} s\left(\varrho_{n}\right)$ is a closed projection for all $m$. Then there is a maximal associative subalgebra $B$ of $A$ such that all states $\left(\varrho_{n}\right)$ restrict to pure states on $B$.

In particular, for a finite system of orthogonal pure states on $A$ with $\sigma$-finite central covers there is always a maximal associative subalgebra such that all states are pure on it.

This result is sharper than existing results even in case of $C^{*}$-algebras [1, 2 , 3, 4, 5, 7, 8]. However, the main difficulty lies in the Jordan case when we have to develop new methods to overcame the lack of Hilbert-space representations which are ubiquitous in $C^{*}$-case.

Summing it up, the question on multiplicativity of an infinite sequence of orthogonal pure states is more or less solved. Nevertheless, the following well known question is still open: Does every pure state on a JB algebra restrict to a pure state on some maximal associative subalgebra? 
The results on the determining elements and multiplicativity of orthogonal pure states have been applied to studying the structure of the compact JB algebras [14] and can also be of some interest for the pure extension property studied e.g. in 9 .

Acknowledgement: The author would like to express his gratitude to the Alexander von Humboldt Foundation, Bonn, for supporting his research. He also acknowledges the support of the Grant Agency of the Czech Republic, Grant no. 201/03/0455, "Noncommutative measure theory", and the support of the Czech Technical University, Grant. No. MSM 210000010, "Applied Mathematics in Technical Sciences".

\section{References}

[1] J.F.Aaarnes, R.V.Kadison: Pure states and approximate identities, Proc. Amer. Math. Soc. 21, (1969), 749-752.

[2] C.A.Akemann: Approximate units and maximal abelian $C^{*}$ - subalgebras, Pacific Journal of Mathematics, Vol 33, No. 3, (1970), 543-550.

[3] C.A.Akemann, J.Anderson, G.K.Pedersen: Excising states on $C^{*}$-algebras, Can. J. Math., Vol. XXXVIII, No. 5, (1986), 1239-1260.

[4] C.A.Akemann, J.Anderson, G.K.Pedersen: Approaching infinity in $C^{*}$ algebras, J. Oper. Theory, 21, (1989), 255-271.

[5] J.Anderson: Extensions, restrictions, and representations of states on $C^{*}$ algebras, Trans. Amer. Math. Soc., Vol. 249, No. 2, (1979), 383-400.

[6] J.Anderson: Pathalogy in the Calkin algebra, J. Operator Theory 2 (1979), 159-167.

[7] B.A.Barnes: Pure states with the restriction property, Proc. Amer. Math. Soc., Vol 33, Number 2, June (1972), 277-294.

[8] J.Bunce: Characters on singly generated $C^{*}$-algebras, Proc. Amer. Math. Soc., 25, (1970), 297-303. 
[9] L.J.Bunce, C.-H. Chu: Unique extension of pure states of $C^{*}$-algebras, J. Operator Theory, 39, (1998), 319-338.

[10] K.R.Davidson: $C^{*}$-Algebras by Example, Fields Institute Monographs, Amer. Math. Soc. (1996).

[11] J.Glimm: A Stone-Weierstrass theorem for $C^{*}$-algebras, Annals of Mathematics, Vol. 72, No. 2, September (1960), 216-244.

[12] H.Hanche-Olsen, E.Stormer: Jordan operator algebras, Pitman Publishing, Boston, London, Melbourne, (1984).

[13] J.Hamhalter: Restricting pure states on JB algebras to maximal associative subalgebras, Tatra Mountains Math. Publ., 15, (1998), 599-607.

[14] J.Hamhalter: Supporting sequences of pure states on JB algebras, Studia Mathematica 136, (1), (1999), 37-47.

[15] J.Hamhalter: Pure states on Jordan algebras, Mathematica Bohemica, Vol. 126, No.1, (2001), 81-91.

[16] J.Hamhalter: Multiplicativity of extremal positive maps on abelian parts of operator algebras, Journal of Operator Theory, 48, (2002), 1-18.

[17] R.V.Kadison, J.R.Ringrose: Fundamentals of the Theory of Operator Algebras, Vol. I, Academic Press, (1983).

[18] G.K.Pedersen: $C^{*}$-algebras and their Automorphism Groups, Academic Press (1979).

[19] E.Stormer: A characterization of pure states of $C^{*}$-algebras, Proc. Amer. Math. Soc. 19, 1 (1968), 1000-1102.

[20] M.Takesaki:Theory of operator algebras I, Proc. Amer. Math. Soc. 19, (1968), 1100-1102. Springer-Verlag (1979).

Jan Hamhalter, Czech Technical University - El. Eng, Department of Mathematics, Technicka 2, 166 27, Prague 6, Czech Republic. e-mail: hamhalte@math.feld.cvut.cz 